\title{
ON MEROMORPHISMS OF ALGEBRAIC SYSTEMS
}

\author{
JUNJI HASHIMOTO
}

Dedicated to the memory of Professor Tadasi Nakayama

\section{Introduction}

In the present paper by an algebraic system (algebra) $A$ we shall mean a system with a set $F$ of operations $f_{\lambda}:\left(x_{1}, \ldots, x_{n}\right) \in A \times \cdots \times A \rightarrow f_{\lambda}\left(x_{1}, \ldots\right.$, $\left.x_{n}\right) \in A$. A polynomial $p\left(x_{1}, \ldots, x_{r}\right)$ is a function of variables $x_{1}, \ldots, x_{r}$ which is either one of the $x_{i}$, or (recursively) a result of some operation $f_{\lambda}\left(p_{1}, \ldots\right.$, $p_{n}$ ) performed on other polynomials $p_{i}$. An algebra $A$ may satisfy a set $R$ of identities $p\left(x_{1}, \ldots, x_{r}\right)=q\left(x_{1}, \ldots, x_{s}\right)$, and then $A$ shall be called an $(F, R)$-algebra.

By a meromorphism between two algebras admitting the same operations, we mean a many-many correspondence of elements which preserves all algebraic combinations. If $\varphi$ is a meromorphism of $A$ onto $B$, under which the correspondence of elements shall be written $a \rightarrow b(\varphi)$ or $a \varphi b$, then $a_{i} \varphi b_{i}(i=1, \ldots, n)$ imply $f_{\lambda}\left(a_{1}, \ldots, a_{n}\right) \varphi f_{\lambda}\left(b_{1}, \ldots, b_{n}\right)$. We shall write $b \bar{\varphi} a$ to mean $a \varphi b$, and then $\bar{\varphi}$ becomes a meromorphism of $B$ onto $A$. Let $\varphi$ and $\phi$ be meromorphisms from $A$ onto $B$ and from $B$ onto $C$ respectively, and define $a \varphi \psi c$ to mean $a \varphi b$ and $b p c$ for some $b \in B$. Then $\varphi \psi$ becomes a meromorphism from $A$ onto $C$.

Now on a meromorphism of any algebra the following theorem similar to the Homomorphism Theorem holds.

Meromorphism Theorem. Let $\varphi$ be a meromorphism of $A$ onto $B$. If we define the relation $\varphi^{*}$ in $A$ by

$a \varphi^{*} a^{\prime}$ means that for some finite number of elements $a_{0}, a_{1}, \ldots, a_{n} \in A$ and $b_{1}, \ldots, b_{n} \in B$,

$$
a_{0}=a, a^{\prime}=a_{n}, a_{i-1} \varphi b_{i}, a_{i} \varphi b_{i} \quad(i=1, \ldots, n),
$$

then $\varphi^{*}$ is a congruence relation on $A$, and similarly $\bar{\varphi}^{*}$ is that on $B$. Further their homomorphic images are isomorphic: $\varphi^{*}(A) \cong \bar{\varphi}^{*}(B)$.

Received June 30, 1965. 
If, given $b \in B,\{x ; x \varphi b\}$ is necessarily a congruence class under $\varphi^{*}$ in the above theorem and, given $a \in A,\{y ; a \varphi y\}$ is necessarily that under $\bar{\varphi}^{*}$, then $\varphi$ is called a class-meromorphism. As is already known, a meromorphism $\varphi$ is a class-meromorphism if and only if $a \varphi b, a^{\prime} \varphi b$ and $a^{\prime} \varphi b^{\prime}$ imply $a \varphi b^{\prime}$. When $\varphi$ and $\psi$ are two meromorphisms of $A$ onto $B$, we define $\varphi \leqq \psi$ to mean that $a \varphi b$ implies $a \psi b$. Then the above condition that $\varphi$ be a class-meromorphism is written $\varphi \bar{\varphi} \varphi \leqq \varphi$.

In Shoda's theory for abstract algebraic systems the following condition on an algebra $A$ is often assumed:

( $\alpha$ ) Every meromorphism between two homomorphic images of $A$ is a class-meromorphism.

In the present paper we shall deal with meromorphisms of an algebra $A$ onto itself. We shall first show in $\S 2$ that the above condition $(\alpha)$ is equivalent to the condition

( $\beta$ ) Every meromorphism of $A$ onto itself is a class-meromorphism.

A meromorphism $\varphi$ of $A$ onto itself may be regarded as a relation between elements of $A$. If $\varphi$ is reflexive, i.e. $a \varphi a$ holds for all $a \in A$, we shall call $\varphi$ a quasi-congruence. We shall show that a quasi-congruence on $A$ is a classmeromorphism if and only if it is a congruence relation. We shall inquire in $\S 2$ mainly into the symmetricity and transitivity of quasi-congruences in abstract algebras, and discuss the connections among the transitivity, symmetricity and permutability of quasi-congruences.

In $\S 3$ and $\S 4$ we shall deal with quasi-congruences on some real algebraic systems. Especially we shall discuss in $\S 3$ the conditions that quasi-congruences on a semigroup be symmetric and in $\S 4$ that quasi-congruences on a lattice be transitive. The lattice of quasi-congruences on a lattice is not necessarily distributive. We shall lastly give some sufficient conditions for that lattice to be distributive.

\section{Meromorphisms of an abstract algebra onto itself}

Let $\varphi$ and $\psi$ be homomorphisms of $A$ and $\theta$ a meromorphism between $\varphi(A)$ and $\psi(A)$. If we define $a \Theta b$ to mean $\varphi(a) \theta \psi(b)$, then it is easy to see that $\Theta$ is a meromorphism of $A$ onto itself. Suppose that $\varphi(a) \theta \psi(b), \varphi\left(a^{\prime}\right) \theta \psi(b)$ and $\varphi\left(a^{\prime}\right) \theta \varphi\left(b^{\prime}\right)$. Then $a \Theta b, a^{\prime} \Theta b$ and $a^{\prime} \Theta b^{\prime}$; hence if $\Theta$ is a class-meromorphism 
we get $a \Theta b^{\prime}$ and $\varphi(a) \theta \psi\left(b^{\prime}\right)$, which shows that $\theta$ is a class-meromorphism between $\varphi(A)$ and $\psi(A)$. Thus we have

THeorem 2.1. Every meromorphism between two homomorphic images of an algebra $A$ is a class-meromorphism if and only if every meromorphism of $A$ onto itself is a class-meromorphism.

Meromorphisms of $A$ onto itself form a partially ordered semigroup $M(A)$ under the multiplication and the ordering defined in $\S 1$ :

$$
\begin{aligned}
& a \varphi \psi b \text { means that } a \varphi c \text { and } c \psi b \text { for some } c \in A \text {; } \\
& \varphi \leqq \psi \text { means that } a \varphi b \text { implies } a \psi b .
\end{aligned}
$$

Further, it is rather evident that $\varphi \leqq \varphi_{1}$ and $\psi \leqq \psi_{1}$ imply $\varphi \psi \leqq \varphi_{1} \psi_{1}$.

A meromorphism $\theta$ of $A$ onto itself is regarded as a relation in $A$, and it becomes a congruence relation if it is reflexive, symmetric (symbolically $\bar{\theta} \leqq \theta$ ) and transitive $\left(\theta^{2} \leqq \theta\right)$. A quasi-congruence on $A$ is a meromorphism of $A$ onto itself which is reflexive. The set $Q(A)$ of quasi-congruences on $A$ becomes a subsemigroup of $M(A)$ mentioned above and a complete lattice under the ordering defined in $M(A)$. In $Q(A) a \rightarrow b\left(\Lambda_{\alpha} \theta_{\alpha}\right)$ means that $a \theta_{\alpha} b$ for all $\theta_{\alpha}$.

Now let $P$ be a set of ordered pairs $(a, b)$ of elements of $A$, and define the relation $\theta$ in the following way:

$u \theta v$ means that a polynomial $p\left(x_{1}, \ldots, x_{m}, y_{1}, \ldots, y_{n}\right)$ exists such that

$$
\begin{array}{r}
u=p\left(a_{1}, \ldots, a_{m}, c_{1}, \ldots, c_{n}\right) \text { and } v=p\left(b_{1}, \ldots, b_{m}, c_{1}, \ldots, c_{n}\right) \\
\\
\quad \text { for some }\left(a_{i}, b_{i}\right) \in P .
\end{array}
$$

Then it is easily seen that $\theta$ becomes a quasi-congruence, which is the least of elements $\varphi$ of $Q(A)$ satisfying $a \varphi b$ for every pair $(a, b) \in P$. This $\theta$ is called the quasi-congruence generated by $P$ and denoted by $\theta(P)$. It follows that $\theta(P)=V_{i a, b j \in P} \theta(a, b)$, where $\theta(a, b)$ is the quasi-congruence generated by one pair $(a, b)$.

We intend to discuss the symmetricity and transitivity of quasi-congruences. We first show

Theorem 2.2. Let $\left\{\theta_{\alpha}\right\}$ be a set of quasi-congruences on an algebra $A$. Then $\overline{\Lambda_{\alpha} \theta_{\alpha}}=\Lambda_{\alpha} \bar{\theta}_{\alpha}$ and $\overline{V_{\alpha} \theta_{\alpha}}=V_{\alpha} \bar{\theta}_{\alpha} ;$ accordingly symmetric quasi-congruences form a closed sublattice of $Q(A)$. 
Proof. It is clear by the meaning that $\overline{\Lambda_{\alpha} \theta_{\alpha}}=\Lambda_{\alpha} \bar{\theta}_{\alpha}$. Let $P$ be a set of ordered pairs $(a, b)$ of elements of $A$ and put $\bar{P}=\{(b, a) ;(a, b) \in P\}$. If $u \rightarrow v(\theta(P))$, then a polynomial $p$ exists such that $u=p\left(a_{1}, \ldots, a_{m}, c_{1}, \ldots, c_{n}\right)$, $v=p\left(b_{1}, \ldots, b_{m}, c_{1}, \ldots, c_{n}\right)$ and $\left(a_{i}, b_{i}\right) \in P$. Then $\left(b_{i}, a_{i}\right) \in \bar{P}$ and hence we infer $v \rightarrow u(\theta(\bar{P}))$, which shows $\overline{\theta(P)}=\theta(\bar{P})$. Now put $\theta_{\alpha}=\theta\left(P_{\alpha}\right)$. Then $V_{\alpha} \theta_{\alpha}=\theta\left(V_{\alpha} P_{\alpha}\right)$, where $V_{\alpha} P_{\alpha}$ is the set-sum of $P_{\alpha}$. So we can deduce

$$
\overline{V_{\alpha} \theta_{\alpha}}=\overline{\theta\left(V_{\alpha} P_{\alpha}\right)}=\theta\left(\overline{V_{\alpha} P_{\alpha}}\right)=\theta\left(V_{\alpha} \bar{P}_{\alpha}\right)=V_{\alpha} \theta\left(\bar{P}_{\alpha}\right)=V_{\alpha} \bar{\theta}_{\alpha},
$$

completing the proof.

If quasi-congruences $\theta_{\alpha}$ are transitive, then $\Lambda_{\alpha} \theta_{\alpha}$ is also transitive but $V_{\alpha} \theta_{\alpha}$ is not necessarily transitive; hence the set $\Theta(A)$ of congruences on $A$ is meetclosed in $Q(A)$ but not always a sublattice of $Q(A)$.

Now let $S$ be a subalgebra of an algebra $A$ and every quasi-congruence on $S$ be transitive. Suppose $x, y, z \in S, x \theta y$ and $y \theta z$ under a quasi-congruence $\theta$ on $A$. Since $\theta$ can be regarded as a quasi-congruence $\theta_{0}$ on $S$, provided the range of elements is restricted in $S$, and $\theta_{0}$ is transitive, we infer $x \theta_{0} z$ and $x \theta z$. So we have

Theorem 2.3. Quasi-congruences on an algebra $A$ are transitive if every triple $\{x, y, z\}$ is contained in a subalgebra $S=S(x, y, z)$ on which quasi-congruences are transitive.

And similarly,

THEOREM 2.4. Quasi-congruences on an algebra $A$ are symmetric if every pair $\{x, y\}$ is contained in a subalgebra $S=S(x, y)$ on which quasi-congruences are symmetric.

Two quasi-congruences $\varphi$ and $\psi$ are called permutable if and only if $\varphi \psi=$ $\phi \varphi$. We see some connections among the transitivity, symmetricity and permutability of quasi-congruences.

TheOREM 2.5. If the join $\varphi \cup \psi$ of two quasi-congruences $\varphi$ and $\psi$ is transitive, then $\varphi \psi=\psi \varphi=\varphi \cup \psi$.

Proof. When $\varphi$ and $\psi$ are quasi-congruences on $A, a \varphi b$ implies $a \varphi b \psi b$; hence we have $\varphi \leqq \varphi \psi, \phi \leqq \varphi \psi$ and $\varphi \cup \psi \leqq \varphi \psi$. So we can deduce from $(\varphi \cup \psi)^{2}$ $\leqq \varphi \cup \psi, \varphi \psi \leqq(\varphi \cup \psi)^{2} \leqq \varphi \cup \psi \leqq \varphi \psi$. 
TheоRem 2.6. If quasi-congruences $\varphi, \psi$ and $\varphi \psi$ are symmetric, then $\varphi$ and $\phi$ are permutable.

Proof. It is easily seen that $\overline{\varphi \psi}=\bar{\psi} \bar{\varphi}$. Hence the symmetricity implies $\varphi \psi$ $=\overline{\varphi \psi}=\bar{\phi} \bar{\varphi}=\psi \varphi$.

Next we deal with congruence relations regarded as quasi-congruences. Given a quasi-congruence $\theta$, it follows from the Meromorphism Theorem mentioned in $\S 1$ that $\theta^{*}=V_{n}(\theta \bar{\theta})^{n}$ is a congruence, which is called generated by $\theta$, and if $\theta$ is originally a congruence, $\theta^{*}=\theta$.

TheOREM 2.7. A quasi-congruence is a class-meromorphism if and only if it is a congruence.

Proof. If $\theta$ is a congruence on $A$, then $\theta=V_{n}(\theta \bar{\theta})^{n} \geqq \theta \bar{\theta} \theta \bar{\theta} \geqq \theta \bar{\theta} \theta$, whence $\theta$ is a class-meromorphism. Conversely if $\theta \bar{\theta} \theta \leqq \theta$, then $\bar{\theta} \leqq \theta \bar{\theta} \theta \leqq \theta$ and $\theta^{2} \leqq \theta \bar{\theta} \theta \leqq \theta$; hence $\theta$ is a congruence.

The set $\Theta(A)$ of congruences on $A$ is not always a sublattice or a subsemigroup of $Q(A)$. We shall give below some conditions for $\Theta(A)$ to be so.

The product $\varphi \psi$ of two congruences $\varphi$ and $\psi$ becomes a congruence if and only if they are permutable; hence

Theorem 2.8. Congruences on an algebra $A$ form a subsemigroup of $Q(A)$ if and only if they are permutable.

Let $\varphi$ and $\psi$ be congruences on $A$ and $\varphi \vee \psi$ the congruence generated by $\varphi \psi$. Then $\varphi \cup \psi \leqq \varphi \psi \leqq \varphi \vee \psi$. Hence we can infer from Theorem 2.5,

TheOREm 2.9. If quasi-congruences on an algebra $A$ are transitive, then congruences on $A$ form a sublattice of $Q(A)$. If congruences on $A$ form a sublattice of $Q(A)$, then they are permutable.

As shown above the transitivity or symmetricity of quasi-congruences implies the permutability of congruences. Hence if quasi-congruences are class-meromorphisms, then congruences are permutable. But the converse is not true. On the other hand Malcev [2] has proved the following theorem.

THEOREM 2.10 (Malcev). If congruences on every $(F, R)$-algebra are permutable, then there exists a polynomial $p(x, y, z)$ such that $p(x, y, y)=x$ and $p(x, x, y)=y$. 
If such a polynomial $p(x, y, z)$ exists, then $a \varphi b, a^{\prime} \varphi b$ and $a^{\prime} \varphi b^{\prime}$ imply $a=$ $p\left(a, a^{\prime}, a^{\prime}\right) \varphi p\left(b, b, b^{\prime}\right)=b^{\prime}$. Hence

TheоRem 2.11. If congruences on every $(F, R)$-algebra are permutable, then meromorphisms of every $(F, R)$-algebra onto itself are class-meromorphisms.

\section{Quasi-congruences on a semigroup}

We intend to obtain the condition for a semigroup $G$ that every quasicongruence on $G$ be a congruence. We have succeeded to solve this problem for a commutative semigroup.

Theоrem 3.1. For a commutative semigroup $G$ the following conditions are equivalent:

(1) every quasi-congruence on $G$ is symmetric,

(2) $G$ is a group in which every element has a finite order.

Proof. (1) $\rightarrow(2)$. Let $a$ be any element of $G$. If we define $x \theta y$ to mean either $x=y$ or $x=y a^{n}$ with $n=1,2, \ldots$, then it is easy to see that $\theta$ is a quasi-congruence on $G$. Since $a^{2} \theta a$ and $\theta$ is symmetric, we get $a \theta a^{2}$ and $a=$ $a^{n+1}(n=1,2, \ldots)$. Put $e=a^{n}$. If $n=1$, then $e^{2}=a^{2}=a=e$, and if $n \geqq 2$, then $e^{2}=a^{n+1} a^{n-1}=a a^{n-1}=a^{n}=e$. Since $e x \theta x$, we have $x \theta e x$, that is either $x=e x$ or $x=e x a^{n}$, and then we can show $e x=x$ by $e^{2}=e$; namely $e$ is an identity. Similarly, given $b \in G$, we can find $e^{\prime}=b^{m}$ such that $e^{\prime} x=x$ for all $x \in G$, and then we have $e^{\prime}=e e^{\prime}=e^{\prime} e=e$ and either $b=e$ or $b^{m-1} b=e$; so $b$ has an inverse and a finite order.

Now the implication $(2) \rightarrow(1)$ can be shown without the commutativity of G. Namely

TheоReм 3.2. If $G$ is a group in which every element has a finite order, then every quasi-congruence $\theta$ on $G$, regarded as a semigroup, is a congruence.

Proof. $a \theta b$ and $b \theta c$ imply $a b^{-1} b \theta b b^{-1} c$, that is $a \theta c$. Hence every quasicongruence on a group is transitive. Suppose that $a \theta b$ and the order of $c=a b^{-1}$ is $n$. If $n=1$, then $a=b$ and $b \theta a$. If $n \geqq 2$, then $c=a b^{-1} \theta 1$ implies $c^{-1}=$ $c^{n-1} \theta 1$ and $b a^{-1} \theta 1$; whence we get $b \theta a$. Thus $\theta$ is a congruence.

As is already known, a congruence $\theta$ on a group $G$ regarded as a semigroup becomes that on $G$ regarded as a group; namely preserves the operation $f(x)$ $=x^{-1}$. On the other hand every meromorphism between groups, preserving 
$f(x)=x^{-1}$, is a class-meromorphism. Hence Theorem 3.1. shows that a quasicongruence on a group $G$ regarded as a semigroup is not necessarily that on $G$ regarded as a group and further the permutability of quasi-congruences on a semigroup does not imply the symmetricity of those.

\section{Quasi-congruences on a lattice}

In the present section we intend to discuss the properties of quasi-congruences on a lattice with the operations $U$ and $\cap$. A semilattice on which quasi-congruences are symmetric is trivial. For every element of a semilattice $L$, regarded as a commutative semigroup under the multiplication $U$, is idempotent, and so $L$ can contain no element other than one element 1 if it forms a group. This follows also from the fact that the relation $\leqq$ becomes a quasicongruence in a semilattice or a lattice; hence

TheоRem 4.1. Some quasi-congruence on a lattice (semilattice) $L$ is not symmetric, provided L contains two or more elements.

Then we consider the transitivity of quasi-congruences on a lattice $L$.

Lemma 4.1. Let $\theta$ be a quasi-congruence on a lattice $L$. If the implication $a \theta b \theta c \rightarrow a \theta c$ holds for the cases $a \leqq b \leqq c$ and $a \geqq b \geqq c$, then $\theta^{2}=\theta$.

Proof. $\quad a \theta b \theta c$ implies $a \cup a \theta a \cup b, a \cup b \cup b \theta a \cup b \cup c$ and $a \theta a \cup b \cup c$, since $a \leqq$ $a \cup b \leqq a \cup b \cup c$. Similarly $a \cup b \cup c \theta b \cup c \theta c$ implies $a \cup b \cup c \theta c$. Then we have $a \cap(a \cup b \cup c) \theta(a \cup b \cup c) \cap c$, that is $a \theta c$.

Now we call an element $m$ of a lattice modular if $x \leqq y$ implies $x \cup(m \cap y)$ $=(x \cup m) \cap y$.

Theorem 4.2. Let $m$ be a modular element in a lattice $L$. If all intervals containing $m$ are complemented, then quasi-congruences on $L$ are transitive.

Proof. We shall show for $a \leqq b \leqq c$ that $a \theta b \theta c$ implies $a \theta c$. Let $x$ be a relative complement of $b \cup m$ in the interval $[a \cap m, c \cup m]$ and $y$ that of $(b \cup x) \cap m$ in $[a \cap m, m]$. Then we get

$$
\begin{aligned}
& a=a \cup(a \cap m)=a \cup(x \cap(b \cup m)) \theta b \cup(x \cap(c \cup m))=b \cup x, \\
& y=(a \cap m) \cup y \theta((b \cup x) \cap m) \cup y=m
\end{aligned}
$$

and 


$$
\begin{aligned}
& a=a \cup(a \cap m)=a \cup(y \cap((b \cup x) \cap m))=a \cup(y \cap(b \cup x)) \theta \\
& (b \cup x) \cup(m \cap(c \cup x))=(b \cup x \cup m) \cap(c \cup x)=(c \cup m) \cap(c \cup x) ;
\end{aligned}
$$

accordingly $c \cap a \theta c \cap(c \cup m) \cap(c \cup x)$, that is $a \theta c$.

Dually we can show that $a \geqq b \geqq c$ and $a \theta b \theta c$ imply $a \theta c$. Hence it follows from Lemma 4.1 that $\theta$ is transitive.

A lattice with 0 in which all intervals $[0, x]$ are complemented is called section-complemented. For a lattice $L$ without 0 we shall define $L$ to be sectioncomplemented when every element of $L$ is contained in a section-complemented principal dual ideal. If a lattice $L$ is section-complemented, then any triple $\{x, y, z\}$ is contained in a section-complemented dual ideal $S=[a)$, in which the condition in Theorem 4.2 holds; hence by Theorem 2.3 we infer

COROLlaRY 1. In a section-complemented lattice every quasi-congruence is transitive.

Further, by Theorem 2.5 we can assert the following propositions in our previous paper [1].

Corollary 2. If all intervals of a lattice $L$ containing a modular element $m$ are complemented, then congruence relations on $L$ are permutable.

Corollary 3. On a section-complemented lattice congruence relations are permutable.

Next we shall inquire into the structure of the lattice $Q(L)$ of quasicongruences on a lattice $L$. It is well-known that congruence relations on a

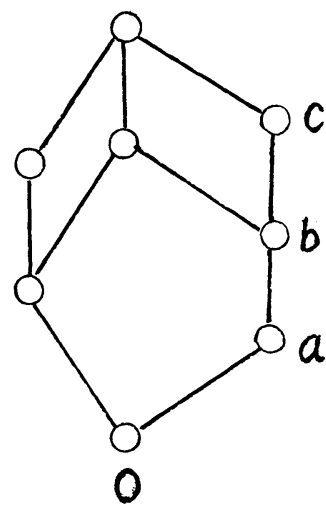

FIG. 1 
lattice form a distributive lattice. However the lattice $Q(L)$ is not necessarily modular. Indeed if we set in the lattice of Fig. 1

$$
\theta=\theta(0, b), \varphi=\theta(b, c) \text { and } \phi=\theta(a, c),
$$

then $\varphi \leqq \psi$ and $a \rightarrow c((\varphi \cup \theta) \cap \psi)$ holds nevertheless $a \rightarrow c(\varphi \cup(\theta \cap \psi))$ does not hold.

Lemma 4.2. If we define in a lattice $L a \omega b$ to mean $a \leqq b$, then $\omega$ is a quasicongruence on $L$ and a lower distributive element in $Q(L): \omega \cap(\varphi \cup \psi)=(\omega \cap \varphi)$ $\cup(\omega \cap \psi)$ for all $\varphi, \psi \in Q(L)$.

Proof. Put $\rho=\omega \cap(\varphi \cup \psi), \varphi_{0}=\omega \cap \varphi, \psi_{0}=\omega \cap \psi$ and $\sigma=\varphi_{0} \cup \psi_{0}$. It suffices to show $\rho \leqq \sigma$. As is mentioned in $\S 1$, x $\rho y$ implies that a lattice polynomial $p$ exists such that

$$
\begin{aligned}
& x=p\left(a_{1}, \ldots, a_{l}, s_{1}, \ldots, s_{m}, u_{l}, \ldots, u_{n}\right), \\
& y=p\left(a_{1}, \ldots, a_{l}, t_{1}, \ldots, t_{m}, v_{1}, \ldots, v_{n}\right)
\end{aligned}
$$

and $x \leqq y, s_{i} \varphi t_{i}, u_{j} \psi v_{j}$. Then since $s_{i} \varphi s_{i} \cup t_{i}$ and $u_{j} \psi u_{j} \cup v_{j}$, we get $s_{i} \varphi_{c} s_{i} \cup t_{i}$ and $u_{j} \psi_{0} u_{j} \cup v_{j}$. Hence if we put

$$
z=p\left(a_{1}, \ldots, a_{l}, s_{1} \cup t_{1}, \ldots, s_{m} \cup t_{m}, u_{1} \cup v_{1}, \ldots, u_{n} \cup v_{n}\right),
$$

then we get $x \leqq y \leqq z, x_{\sigma} z$ and $x=x \cap y_{\sigma} \cap y=y$, proving $\rho \leqq \sigma$.

Dually we define $a \omega^{\prime} b$ to mean $a \geqq b$. Then we can show

Lemma 4.3. If $\theta \cap(\varphi \cap \psi)=(\theta \cap \varphi) \cup(\theta \cap \psi)$ holds for the cases $\theta, \varphi, \psi \leqq \omega$ and $\theta, \varphi, \psi \leqq \omega^{\prime}$ in $Q(L)$, then $Q(L)$ is distributive.

Proof. Let $\theta, \varphi$ and $\psi$ be any quasi-congruences on $L$ and put $\rho=\theta \cap(\varphi$ $\cup \psi), \sigma=(\theta \cap \varphi) \cup(\theta \cap \psi)$. Then by Lemma 4.2 we get $\omega \cap \rho=(\omega \cap \theta) \cap((\omega \cap$ $\varphi) \cup(\omega \cap \psi))$, and by the assumption $\omega \cap \rho=(\omega \cap \theta \cap \varphi) \cup(\omega \cap \theta \cap \psi) \leqq \sigma$. Hence $x \rho y$ implies $x \cap y \rho y, x \cap y(\omega \cap \rho) y$ and $x \cap y \sigma y$. Dually we can show that $x \rho y$ implies $x_{\sigma} x \cap y$. Then we have $(x \cap y) \cup x_{\sigma} y \cup(x \cap y)$, $x_{\sigma} y$ and thus $\rho \leqq \sigma$.

Theorem 4.3. If all quasi-congruences on a lattice are transitive, then they form a distributive lattice.

Proof. By Lemma 4.3, it is sufficient to prove $\theta \cap(\varphi \cup \psi)=(\theta \cap \varphi) \cup(\theta \cap \psi)$ for $\theta, \varphi, \phi \leqq \omega$. Put $\rho=\theta \cap(\varphi \cup \psi)$ and $\sigma=(\theta \cap \varphi) \cup(\theta \cap \psi)$. Since $\sigma$ is transitive, we can write $\sigma=(\theta \cap \varphi)(\theta \cap \psi)$ by Theorem 2.5. If $x \rho y$, then we have

$$
x=p\left(a_{1}, \ldots, a_{l}, s_{1}, \ldots, s_{m}, u_{1}, \ldots, u_{n}\right),
$$




$$
y=p\left(a_{1}, \ldots, a_{l}, t_{1}, \ldots, t_{m}, v_{1}, \ldots, v_{n}\right)
$$

with $s_{i} \varphi t_{i}, u_{j} \psi v_{j}$. If we put

$$
z=p\left(a_{1}, \ldots, a_{l}, t_{1}, \ldots, t_{m}, u_{1}, \ldots, u_{n}\right),
$$

then $x \varphi z, z \psi y$ and $x \leqq z \leqq y$, since $\varphi, \psi \leqq \omega$. Since $x \theta y, x=x \cap z \theta y \cap z=z$ and $z=$ $x \cup z \theta y \cup z=y$. Hence we have $x(\theta \cap \varphi) z, z(\theta \cap \psi) y$ and $x(\theta \cap \varphi)(\theta \cap \psi) y$; namely $x \sigma y$. Thus $\theta \cap(\varphi \cup \psi)=(\theta \cap \varphi) \cup(\theta \cap \psi)$.

COROLlary. The lattice of quasi-congruences on a section-complemented lattice is distributive.

THEOREM 4.4. The lattice of quasi-congruences on a distributive lattice is distributive.

Proof. Put $\rho=\theta \cap(\varphi \cup \psi)$ and $\sigma=(\theta \cap \varphi) \cup(\theta \cap \psi)$ for quasi-congruences $\theta$, $\varphi, \psi \leqq \omega$, and assume that $x \mu y$. Then we can write

$$
\begin{aligned}
& x=p(a, s, u)=p\left(a_{1}, \ldots, a_{l}, s_{1}, \ldots, s_{m}, u_{1}, \ldots, u_{n}\right), \\
& y=p(a, t, v)=p\left(a_{1}, \ldots, a_{l}, t_{1}, \ldots, t_{m}, v_{1}, \ldots, v_{n}\right)
\end{aligned}
$$

with $s_{i} \varphi t_{i}, u_{j} \psi v_{j}$. We define two weights $w_{\mathrm{i}}(p)$ and $w_{2}(p)$ of the polynomial $p$ by $w_{1}(p)=m+n$ and $w_{2}(p)=l+m+n$. We shall prove $x \sigma y$ by induction on $w_{1}(p)$ and $w_{2}(p)$. If $w_{1}(p) \geqq 2$, we can write either $p=p_{1} \cap p_{2}$ or $p=p_{1} \cup p_{2}$ with $w_{1}(p)=w_{1}\left(p_{1}\right)+w_{1}\left(p_{2}\right), w_{2}(p)=w_{2}\left(p_{1}\right)+w_{2}\left(p_{2}\right), w_{1}\left(p_{i}\right) \geqq 0$ and $w_{2}\left(p_{i}\right) \geqq 1$. We may deal only with the case $p=p_{1} \cap p_{2}$.

Case 1. $w_{1}\left(p_{1}\right)<w_{1}(p), w_{1}\left(p_{2}\right)<w_{1}(p)$. Since $x \rho y$ and

$$
x \leqq y \cap p_{1}(a, s, u) \leqq y \cap p_{1}(a, t, v)=y,
$$

we get $y \cap p_{1}(a, s, u) \rho y \cap p_{1}(a, t, v)$. Since $w_{1}\left(y \cap p_{1}\right)=w_{1}\left(p_{1}\right)<w(p)$, we get $y \cap p_{1}(a, s, u)_{\sigma y} \cap p_{1}(a, t, v)=y$, by the hypothesis of induction, and similarely $y \cap p_{2}(a, s, u) \sigma y$. Then

$$
x=\left(y \cap p_{1}(a, s, u)\right) \cap\left(y \cap p_{2}(a, s, u)\right)_{\sigma y} .
$$

Case 2. $w_{1}\left(p_{1}\right)=w_{1}(p), w_{1}\left(p_{2}\right)=0$. If we put $b=p_{2}(a)$, then $x=p_{1}(a, s$, $u) \cap b, y=p_{1}(a, t, v) \cap b$ and hence $x=p_{1}(a, s, u) \cap y, y=p_{1}(a, t, v) \cap y$, We can write either $p_{1}=p_{3} \cap p_{4}$ or $p_{1}=p_{3} \cup p_{4}$ in the same manner as above. If $p_{1}=p_{3} \cap p_{4}$, then by regarding $p_{3}$ and $p_{4} \cap b$ as $p_{1}$ and $p_{2}$ we can reduce to either Case 1 or the case $p_{1}=p_{3} \cup p_{4}$. Hence we may assume that $p_{1}=p_{3} \cup p_{4}$.

Case 2.1. $w_{1}\left(p_{3}\right)<w_{1}\left(p_{1}\right), w_{1}\left(p_{4}\right)<w_{1}\left(p_{1}\right)$. Since $x \rho y$ and 


$$
x=\left(p_{3}(a, s, u) \cap y\right) \cup x \leqq\left(p_{3}(a, t, v) \cap y\right) \cup x \leqq y,
$$

we get $\left(p_{3}(a, s, u) \cap y\right) \cup x \rho\left(p_{3}(a, t, v) \cap y\right) \cup x$ and $\left.\left.w_{1}^{\prime}\left(p_{3} \cap y\right) \cup x\right)=w_{1}^{\prime} p_{3}\right)<$ $w_{1}(p)$. Hence we have $x_{\sigma}\left(p_{3}(a, t, v) \cap y\right) \cup x, x_{\sigma}\left(p_{4}(a, t, v) \cap y\right) \cup x$ and $x_{\sigma}\left(p_{3}(a\right.$, $t, v) \cap y) \cup x \cup\left(p_{4}(a, t, v) \cap y\right) \cup x=\left(p_{1}(a, t, v) \cap y\right) \cup x=y$ by the distributivity.

Case 2.2. $w_{1}\left(p_{3}\right)=w_{1}\left(p_{1}\right), w_{1}\left(p_{4}\right)=0$. Then we can write, putting $p_{4}(a)=c$,

$$
\begin{aligned}
& x=\left(p_{3}(a, s, u) \cup c\right) \cap y=\left(p_{3}(a, s, u) \cap y\right) \cup(c \cap y), \\
& y=\left(p_{3}(a, t, v) \cup c\right) \cap y=\left(p_{3}(a, t, v) \cap y\right) \cup(c \cap y)
\end{aligned}
$$

and $x=\left(p_{3}(a, s, u) \cap y\right) \cup x, y=\left(p_{3}(a, t, v) \cap y\right) \cup x$, since $c \cap y \leqq x$. We may assume $p_{3}=p_{5} \cap p_{6}$ without loss of generality. Then since $x_{\rho} y$ and

$$
x \leqq\left(p_{b}(a, s, u) \cap y\right) \cup x \leqq\left(p_{5}(a, t, v) \cap y\right) \cup x=y,
$$

we have $\left(p_{5}(a, s, u) \cap y\right) \cup x \rho\left(p_{5}(a, t, v) \cap y\right) \cup x$. Since $w_{2}\left(\left(p_{5} \cap y\right) \cup x\right)=$ $w_{2}\left(p_{\overline{5}}\right)+2$ and $w_{2}\left(p_{\overline{5}}\right)<w_{2}\left(p_{3}\right)<w_{2}\left(p_{1}\right)<w_{2}\left(p^{\prime}, w_{2}\left(\left(p_{5} \cap y\right) \cup x\right)<w_{2}(p)\right.$. Hence we can infer $\left(p_{5}(a, s, u) \cap y\right) \cup x_{\sigma}\left(p_{5}(a, t, v) \cap y\right) \cup x=y$, by the hypothesis of induction, and $\left(p_{0}(a, s, u) \cap y\right) \cup x \sigma y$. Then

$$
\begin{aligned}
x & =\left(p_{\overline{5}}(a, s, u) \cap p_{6}(a, s, u) \cap y\right) \cup x \\
& =\left(\left(p_{\overline{5}}(a, s, u) \cap y\right) \cup x\right) \cap\left(\left(p_{\dot{5}}(a, s, u) \cap y\right) \cup x\right)_{\sigma} y,
\end{aligned}
$$

completing the proof.

It seems the distributivity of $Q(L)$ may be deduced from more weaker conditions on $L$. For instance we guess that $Q(L)$ may be distributive for a modular lattice $L$. Further we intend to inquire into the structure of a lattice $L$ by the investigation of $Q(L)$ but we have obtained no useful result on it.

\section{References}

[1] J. Hashimoto: Congruence relations and congruence classes in lattices, Osaka Math. J. 15 (1963).

[2] A. I. Malcev: On the general theory of algebraic systems, Mat. Sb. N. S. 35 (77) (1954), Amer. Math. Soc. Transl. (2) 27 (1963).

[3] T. Nakayama: Sets, topologies and algebraic systems (Shugo, Iso, Daisukei in Japanese), Tokyo, 1949.

[4] K. Shoda: Universal theory for algebra (Daisugaku Tsuron in Japanese), Tokyo, 1947.

[5] K. Shoda: Uber die allgemeinen algebraischen Systeme I-VII, Proc. Imp. Acad. Tokyo 17$20(1941-4)$.

\section{Department of Mathematics}

Kobe University 